\title{
Conocimientos, hábitos, niveles de confianza para la resolución de problemas matemáticos en los estudiantes de pedagogía
}

(c) (i)(9)

Knowledge, habits, confidence levels for solving mathematical problems in pedagogy students

\author{
Gonzalo Efrén Hidalgo Portocarrero. ' , Patricio Alejandro Merino Córdova. ${ }^{2}$, Byron \\ Fabricio Estupiñan Cox. ${ }^{3} \&$ Omar Edison Tapia Aguilar. ${ }^{4}$
}

Recibido: 03-09-2021 / Revisado: 15-09-2021 /Aceptado: 21-10-2021/ Publicado: 05-11-2021

\begin{abstract}
DOI: https://doi.org/10.33262/concienciadigital.v4i4.1.1922

Introduction. Mathematics is one of the disciplines that include a series of contents that on many occasions are difficult for students to understand, hindering their learning, and the acquisition of skills and abilities that are necessary in their academic life and in all kinds of social contexts. -educational. Objective. Analyze the knowledge, habits and levels of confidence for solving mathematical problems in students of the Pedagogy of Experimental Sciences of Mathematics and Physics at the Technical University "Luis
\end{abstract}

\footnotetext{
${ }^{1}$ Docente de Matemática - Física de la Unidad Educativa del Milenio Simón Plata Torres del Cantón Esmeraldas. Maestrante de la Maestría en Matemática, Mención Modelación y Docencia, Universidad Técnica Luis Vargas Torres de Esmeraldas, gonzalo.hidalgo.portocarrero@utelvt.edu.ec, ID ORCID: https://orcid.org/0000-0001-7603-693X

2 Docente de la Facultad de ciencias Agropecuarias Carrera de Agronomía. Maestrante de la Maestría en Matemática, Mención Modelación y Docencia. Universidad Técnica Luis Vargas Torres de Esmeraldas, patricio.merino@utelvt.edu.ec, ID ORCID: https://orcid.org/0000-0001-5068-8673

3 Docente de Matemáticas en la Facultad de Ciencias Agropecuarias,Carrera de Ingeniería Forestal, Maestrante de la Maestría en Matemáticas. Mención Modelación y Docencia, Universidad Técnica Luis Vargas Torres de Esmeraldas, byron.estupinan@utelvt.edu.ec, ID ORCID: https://orcid.org/0000-00016523-667X

${ }^{4}$ Maestrante de la Maestría en Matemáticas. Mención Modelación y Docencia. Universidad Técnica Luis Vargas Torres de Esmeraldas, Omar.tapia.aguilar@utelvt.edu.ec, ID ORCID: https://orcid.org/0000-00020922-3046
} 
Vargas Torres" in Esmeraldas. Methodology. It has a quantitative approach, a bibliographic, field and analytical research is applied. The method implemented is the deductive one, the sample used corresponds to 45 students belonging to the Pedagogy career. Results. The results obtained conclude that knowledge, habits and confidence levels influence the resolution of mathematical problems in this group of students; This is because the previous mathematical knowledge that the student possesses allows him to strategically approach the problem that needs to be solved, while habits contribute to the development of skills and abilities necessary in this activity, likewise the level of confidence can contribute In solving mathematical problems, then, if the student feels calm and trusts in their abilities, they show a positive attitude to develop this activity for the benefit of their own personal and academic formation. Conclusion. It is concluded that knowledge, habits and confidence levels influence the resolution of mathematical problems in students of the Pedagogy Career, Experimental Sciences of Mathematics and Physics of the Technical University "Luis Vargas Torres".

Keywords: Knowledge, habits, confidence level, mathematical problems.

\section{Resumen}

Introducción. La Matemática es una de las disciplinas que incluyen una serie de contenidos que en muchas ocasiones resultan de difícil comprensión para los estudiantes, obstaculizando su aprendizaje, y la adquisición de habilidades y destrezas que son necesarias en su vida académica y en toda clase de contextos socio-educativo. Objetivo. Analizar los conocimientos, hábitos y niveles de confianza para la resolución de problemas matemáticos en los estudiantes de la Carrera de la Pedagogía de las Ciencias Experimentales de la Matemática y la Física en la Universidad Técnica "Luis Vargas Torres" de Esmeraldas. Metodología. Tiene un enfoque cuantitativo, se aplica una investigación bibliográfica, de campo y analítica. El método implementando es el deductivo, la muestra utilizada corresponde a 45 estudiantes pertenecientes a la carrera de la Pedagogía. Resultados. Los resultados obtenidos concluyen que los conocimientos, hábitos y niveles de confianza influyen en la resolución de problemas matemáticos en este grupo de estudiantes; esto se debe a que los saberes matemáticos previos que el estudiante posee, le permiten abordar estratégicamente el problema que se necesita solucionar, mientras que los hábitos contribuyen con el desarrollo de habilidades y destrezas necesarias en esta actividad, así mismo el nivel de confianza puede contribuir en la resolución de problemas matemáticos, pues, sí el estudiante se siente calmado y confía en sus capacidades demuestra una actitud positiva para desarrollar esta actividad en beneficio de su propia formación personal y académica. Conclusión. Se concluye que los conocimientos, hábitos y niveles de confianza influyen en la resolución de problemas matemáticos en los estudiantes de la Carrera de la Pedagogía las Ciencias Experimentales de la Matemática y la Física de la Universidad Técnica "Luis Vargas Torres".

Palabras clave: Conocimientos, hábitos, nivel de confianza, problemas matemáticos. 


\section{Introducción}

La Matemática es una de las disciplinas que incluyen una serie de contenidos que en muchas ocasiones resultan de difícil comprensión para los estudiantes, obstaculizando su aprendizaje, y la adquisición de habilidades y destrezas que son necesarias en su vida académica y en toda clase de contextos socio-educativo. Esta ciencia, es muy importante y vital para el desarrollo y funcionamiento de todas las sociedades del mundo, así como esencial en la formación integral de las personas, constituyéndose en elemento presente desde temprana edad. La Matemática es uno de los conocimientos más antiguo que la humanidad ha estudiado e investigado, presente en cada una de las actividades de nuestra vida cotidiana.

"El conocimiento se establece como una actividad que consiste principalmente en la elaboración de significados asociados a la propia experiencia, incluida la experiencia linguística del estudiante con la matemática y con el docente de matemática" (Aliaga et al., 2014, p. 134).

El hábito, es una intersección de conocimientos representada por el paradigma teórico, que responde al qué hacer y el por qué, la capacidad es el cómo hacer y el deseo la motivación es el querer hacer. Para convertir algo en un hábito se requiere de éstos tres elementos. Para hablar de hábitos efectivos es imprescindible referirse a los tres aspectos, donde cada uno de ellos responde a un área importante, sin dejar de lado la cohesión e interacción que debe existir en cada uno de ellos (Huari, 2019, p. 24).

El hábito además se entiende como "una disposición a actuar de una determinada manera, y esa determinación está impulsada por un propósito, es decir, que se obtendrá una determinada clase de resultado si la ocasión es adecuada, aunque dicha determinación tiene condiciones y no es absoluta" (Castaño, 2011, p. 2).

Bahamonde y Vicuña (2011) consideran que, la actitud y confianza que el estudiante sienten hacia sus propias capacidades pueden influir de manera positiva o negativa al momento de solucionar problemas matemáticos, puesto que quienes demuestran actitudes positivas, desafiantes y analíticas pueden enfrentarse con éxito a esta clase de procesos; a diferencia de quienes demuestran lo contrario, y peor aún desconfían de sus propias habilidades al enfrentarse a esta clase de actividades matemáticas.

Por su parte, investigadores como Conejeros et al., (2010) sostienen que la actitud y confianza son conceptos "dinámicos que varían en el tiempo, se desarrollan, se construyen, declinan e incluso vuelven a aparecer en relaciones de más largo plazo, pues las relaciones se transforman en el tiempo, incluyendo el caso de los docentes con sus estudiantes" (p. 32).

Para investigadores como Leal y Bong (2015), una de las actividades que experimenta dificultad en el contexto socio-educativo corresponde a la resolución de problemas matemáticos que contribuye al desarrollo del pensamiento lógico-crítico-reflexivo en los 
estudiantes, debido a distintos factores entre los que se incluye el conocimiento que se tiene respecto a la Matemática y cada uno de sus elementos.

Desde la experiencia de Blanco et al., (2015), la resolución de problemas matemáticos es una actividad compleja que se enfrenta a una serie de contratiempos debido a que en muchas situaciones los estudiantes intentan resolverlos de una manera mecánica, sin tomar en cuenta que en tal acción intervienen su capacidad de análisis, comprensión, razonamiento y aplicación.

Otros autores sostienen que la resolución de problemas matemáticos es una "práctica pedagógica contextualizada en un tiempo limitado; integra el saber-saber, saber-hacer y saber-actuar, promueve el interés y el gusto por la Matemática a través de desarrollar habilidades y destrezas vinculadas" (Leal y Bong, 2015, p. 79).

El presente artículo analiza, los conocimientos, hábitos y niveles de confianza para la resolución de problemas matemáticos en los estudiantes de la Carrera de la Pedagogía de las Ciencias Experimentales de la Matemática y la Física en la Universidad Técnica "Luis Vargas Torres" de Esmeraldas.

\section{Metodología}

El diseño metodológico aplicado en la presente investigación, tiene un enfoque cuantitativo puesto que se trabaja con datos numéricos obtenidos a través de una encuesta. Los tipos de investigación utilizados corresponden a la bibliográfica, de campo y analítica. En el caso del primero de ellos, se lo utiliza para revisar distintas publicaciones acerca de los conocimientos, hábitos y niveles de confianza en la resolución de problemas matemáticos, y de esta manera definir a nivel teórico tales variables.

En el caso de la investigación de campo, ésta se aplica al momento de recolectar información sobre el tema estudiado, acudiendo a los estudiantes que forman parte de la Carrera de la Pedagogía de la Matemática y la Física de la Universidad Técnica "Luis Vargas Torres" de Esmeraldas. En cambio, la investigación analítica se utiliza para analizar los datos obtenidos a través de la encuesta y de esta manera comprender la relación entre las variables estudiadas.

El método implementando corresponde al deductivo a través del cual es posible tomar en consideración las teorías demostradas como científicas para explicar el objeto estudiado (Garcés, 2010), es decir, en este caso se analiza todas aquellas investigaciones sobre conocimientos, hábitos y niveles de confianza para vincularos con la resolución de problemas matemáticos en los estudiantes de la Carrera de la Pedagogía.

La muestra utilizada en la investigación corresponde a un total de 45 estudiantes pertenecientes a la Carrera de la Pedagogía de la Matemática y la Física de la Universidad Técnica "Luis Vargas Torres" de Esmeraldas, a quienes se aplicó una encuesta a través de Google Forms, por la dificultad de realizarlos de forma presencial debido al contexto de pandemia que se vive en todo el mundo, incluyendo el caso de Ecuador. 
La encuesta estuvo integrada por un total de 30 preguntas que fueron distribuidas en tres secciones: Conocimientos de la modelación Matemática, Hábitos de estudio para la Matemática y Niveles de confianza del estudiante para la resolución de problemas, cada pregunta presentó cinco opciones de respuesta clasificadas de la siguiente manera: total desacuerdo, bastante desacuerdo, ni acuerdo - ni desacuerdo, bastante de acuerdo y total acuerdo.

Es importante la relación del estudiante, en aspectos como su nivel de conocimiento, los hábitos que demuestra, así como su confianza para abordar dicha resolución, son claves en su aprendizaje; más aún si se considera el nivel educativo en el que se encuentra y las bases cognitivas que ha logrado consolidar hasta el momento.

Conocimientos:

Todo conocimiento es importante en la aplicación de las actividades cotidianas, este conocimiento estará disponible en cualquier momento de la vida.

En lo que compete al capital mismo de la Matemática, se remite a los contenidos, recursos pedagógicos, conceptos y metodologías que se usan en la resolución de problemas, y que pueden contribuir a despertar el interés, motivación y curiosidad del estudiante por abordar esta clase de actividades, fomentando incluso su propio autoaprendizaje y el trabajo colaborativo (Donoso et al., 2020).

Es por ello que existe una estrecha relación entre los conocimientos, hábitos y niveles de confianza que los estudiantes pueden demostrar al momento de resolver tales problemas matemáticos, pero tomando en consideración los recursos didácticos y metodologías con los que cuentan en su proceso de aprendizaje, y que deben adaptarse y dar respuesta a sus necesidades cognitivas y prácticas en su vida (Naghi, s.f.).

Hábitos:

Los hábitos, son el conjunto de procesos, metodologías, que se utilizan para aprovechar al máximo los conocimientos adquiridos.

En cuanto a los hábitos, Pérez y Beltrán (2011), sostienen que se trata de aquellas acciones de pensamiento que influyen en el estudio de la Matemática y que pueden condicionar la resolución de problemas. Tales hábitos comprenden la capacidad del estudiante para ampliar sus conocimientos, construir y conceptualizar los saberes adquiridos, el esfuerzo que establece para aprender los contenidos, su capacidad de interpretación y evaluación de los resultados que va obteniendo en su formación matemática, la consolidación de un aprendizaje no memorístico, sino reflexivo; así como el uso de esquemas, mapas u otras herramientas que son importantes en la resolución de problemas matemáticos.

Complementando a lo referido por Pérez y Beltrán, otros investigadores como Huari (2019), afirman que los hábitos se definen como aquellas actividades que el estudiante realiza de forma continua y automática en beneficio de su propio aprendizaje, y que si se 
aplican de forma positiva pueden contribuir a afrontar de forma eficiente todos los obstáculos cognitivos, externos e internos que inciden en su formación académica.

Como puede comprenderse en la cita expuesta, el hábito se establece como una herramienta cognitiva fundamental en los procesos formativos de los estudiantes, puesto que les permiten realizar actividades enfocadas en aprender aquellos contenidos específicos de su aprendizaje como puede ser el caso de la resolución de problemas matemáticos, todo ello, a partir de una forma efectiva y aprovechando el ahorro de recursos como el tiempo.

Estos mismos autores sostienen que además uno de los errores más comunes al momento de abordar la resolución de problemas matemáticos corresponde a la falta de una suficiente motivación por parte de los estudiantes, así como el aumento de estrés y ansiedad que se produce debido a la necesidad de obtener una solución inmediata a lo que se ha planteado.

Niveles de confianza:

Zamora (2017), sostiene que el nivel de confianza que los estudiantes demuestran respecto a su formación académica puede influir de forma positiva o negativa en las actividades que llevan a cabo, incluyendo el caso de la resolución de problemas matemáticos. La autora considera que la confianza es un estado psíquico a través del cual el estudiante se siente seguro en sus habilidades y conocimientos para resolver un problema o desarrollar eficientemente aquellas actividades que se plantean dentro de una asignatura.

La confianza contribuye a que el estudiante no sienta temor al momento de solucionar un problema matemático, permitiéndole escuchar atentamente las indicaciones dadas por su maestro, no alterarse al enfrentar dificultades al realizar la actividad, además de sentirse satisfecho y motivado en su proceso de aprendizaje, permitiendo que la adquisición de contenidos sea más fácil de llevar a cabo, y de esta manera evitar errores al respecto.

Asimismo, y como puede comprenderse en los aportes teóricos referenciados hasta el momento, los conocimientos, hábitos y niveles de confianza son aspectos que pueden influir positiva o negativamente en la resolución de problemas matemáticos en los estudiantes. Por esta razón es fundamental que, dentro de las aulas, los docentes se interesen por fortalecer estos aspectos de tal manera que contribuyan con la resolución de esta clase de problemas, y se consolide el desarrollo eficiente del pensamiento matemático.

Resolución de problemas matemáticos:

La resolución de problemas matemáticos se constituye como "una herramienta didáctica potente para desarrollar habilidades entre los estudiantes, además de ser una estrategia de fácil transferencia para la vida, puesto que permite al educando enfrentarse a situaciones y problemas que deberá resolver" (Pérez y Ramírez, 2011, p. 170). Debido a la importancia que conlleva el aprendizaje de la resolución de esta clase de problemas, es 
fundamental que los docentes se interesen por hacer uso de metodologías dinámicas que contribuyan a superar los temores y obstáculos que pueden presentarse al abordar esta clase de actividades en el aula.

Respecto al tema Donoso et al. (2020), manifiestan que la resolución de problemas matemáticos es una actividad en que entran en juego procesos cognitivos de los estudiantes y el capital mismo de la Matemática.

En relación a la resolución de problemas matemáticos Pérez y Ramírez (2011), refieren que el bagaje de conocimientos matemáticos tanto formales como informales que posee el estudiante suponen un punto de partida positivo o negativo para el desarrollo de tal actividad. Esto se debe a que mientras más conocimientos formen parte del estudiante, éste tendrá mayores oportunidades para analizar lo que se le plantea y resolver de forma exitosa el problema formulado, aplicando conceptos y principios matemáticos.

Por su parte Bahamonde y Vicuña (2011), sostienen que la resolución de problemas matemáticos debe entenderse como una estrategia para aprender distintos conceptos, paradigmas y modelos vinculados a la Matemática, razón por la cual debe abordarse como una herramienta educativa, y no como una actividad obligatoria que los estudiantes deben dominar, aunque para ello se haga uso de procesos memorísticos y repetitivos.

Blanco et al., (2015) insisten además que la resolución de problemas es un proceso que debe efectuarse tomando en consideración aspectos emocionales y psicológicos del estudiante, puesto que es necesario que sienta interés respecto a lo que está aprendiendo y la forma en que las destrezas adquiridas pueden contribuir en su formación académica y en otros aspectos de su vida cotidiana.

\section{Resultados}

\section{Conocimientos de la modelación Matemática}

Respecto a la primera pregunta vinculada a si los conocimientos básicos de matemática (conceptos teoremas, procedimientos) ayudan a encontrar la solución del problema, la mayoría de los estudiantes correspondientes al 75\% están bastante de acuerdo con tal afirmación, el 15\% están en bastante desacuerdo, mientras que el 10\% restante manifiestan su total acuerdo, datos que se aprecia en la tabla 1.

\section{Tabla 1}

Los conocimientos básicos de matemática ayudan a encontrar la solución del problema

\begin{tabular}{lll}
\hline Alternativas & Frecuencia & Porcentaje \\
\hline Total, acuerdo & 5 & 10,0 \\
Bastante de acuerdo & 38 & 75,0 \\
Bastante en desacuerdo & 7 & 15,0 \\
Total, desacuerdo & 0 & 0,0 \\
Ninguna & 0 & 0,0 \\
Total & 45 & 100 \\
\hline
\end{tabular}


En la segunda pregunta, el $65 \%$ de los estudiantes está bastante de acuerdo y convencido que estrategias como: ensayo error, ayudan a resolver un problema de matemática, el 15\% están en bastante desacuerdo, el 10\% está en total acuerdo, mientras que el 10\% restante no está ni de acuerdo, ni en desacuerdo, información que se refleja en la tabla 2.

\section{Tabla 2}

Estrategias como el ensayo error ayudan a resolver un problema de matemática

\begin{tabular}{lll}
\hline Alternativas & Frecuencia & Porcentaje \\
\hline Total, acuerdo & 5 & 10,0 \\
Bastante de acuerdo & 28 & 65,0 \\
Bastante en desacuerdo & 7 & 15,0 \\
Total, desacuerdo & & 0,0 \\
Ninguna & 5 & 10,0 \\
Total & 45 & 100 \\
\hline
\end{tabular}

En la tercera pregunta, el $70 \%$ de los estudiantes está bastante de acuerdo que los conocimientos de Matemática Académica inciden en el desarrollo de la Matemática Superior, el $20 \%$ están en total acuerdo, mientras que el 10\% restante está bastante en desacuerdo, información que se refleja en la tabla 3.

Tabla 3

Los conocimientos de Matemática Académica inciden en el desarrollo de la Matemática Superior

\begin{tabular}{lll}
\hline Alternativas & Frecuencia & Porcentaje \\
\hline Total, acuerdo & 6 & 20,0 \\
Bastante de acuerdo & 35 & 70,0 \\
Bastante en desacuerdo & 4 & 10,0 \\
Total, desacuerdo & 0 & 0,0 \\
Ninguna & 0 & 0,0 \\
Total & 45 & 100 \\
\hline
\end{tabular}

En la cuarta pregunta, el $60 \%$ de los estudiantes está en total acuerdo que la estrategia matemática que utiliza lo ayuda cuando tiene que resolver problemas de matemática, el $25 \%$ está bastante de acuerdo, el $10 \%$ está en bastante desacuerdo, mientras que el $5 \%$ restante no está ni de acuerdo, ni en desacuerdo, datos que se refleja en la tabla 4.

\section{Tabla 4}

La estrategia matemática que utiliza lo ayuda a resolver problemas de matemática

\begin{tabular}{lll}
\hline Alternativas & Frecuencia & Porcentaje \\
\hline Total, acuerdo & 30 & 60,0 \\
Bastante de acuerdo & 12 & 25,0 \\
Bastante en desacuerdo & 3 & 10,0 \\
Total, desacuerdo & 0 & 0,0 \\
Ninguna & 2 & 5,0 \\
Total & 45 & 100 \\
\hline
\end{tabular}


En la quinta pregunta, el $50 \%$ de los estudiantes está en total desacuerdo que los problemas matemáticos tienen una sola forma de resolución, el 30\% está bastante de acuerdo, mientras que el $20 \%$ restante no está ni de acuerdo, ni en desacuerdo, datos que se refleja en la tabla 5 .

\section{Tabla 5}

Los problemas matemáticos tienen una sola forma de resolución

\begin{tabular}{lll}
\hline Alternativas & Frecuencia & Porcentaje \\
\hline Total, acuerdo & 0 & 0,0 \\
Bastante de acuerdo & 15 & 30,0 \\
Bastante en desacuerdo & 0 & 0,0 \\
Total, desacuerdo & 25 & 50,0 \\
Ninguna & 10 & 20,0 \\
Total & 45 & 100 \\
\hline
\end{tabular}

En la sexta pregunta, el $65 \%$ de los estudiantes está bastante de acuerdo que su capacidad para resolver un problema de matemática, se basa en la estrategia que toma para su solución, el $15 \%$ está en total desacuerdo, el 10\% está en total acuerdo, mientras que otro $10 \%$ restante no está ni de acuerdo, ni en desacuerdo, datos que se refleja en la tabla 6.

\section{Tabla 6}

La capacidad para resolver un problema de matemática se basa en la estrategia

\begin{tabular}{lll}
\hline Alternativas & Frecuencia & Porcentaje \\
\hline Total, acuerdo & 4 & 10,0 \\
Bastante de acuerdo & 31 & 65,0 \\
Bastante en desacuerdo & 0 & 0,0 \\
Total, desacuerdo & 6 & 15,0 \\
Ninguna & 4 & 10,0 \\
Total & 45 & 100 \\
\hline
\end{tabular}

En la séptima pregunta, el $50 \%$ de los estudiantes está en total desacuerdo que mientras más estrategias conoce para resolver un problema de matemática, más se demora en determinar su solución, el $25 \%$ está bastante de acuerdo, el $20 \%$ está bastante en desacuerdo, mientras que el 5\% restante no está ni de acuerdo, ni en desacuerdo, datos que se refleja en la tabla 7.

\section{Tabla 7}

Mientras más estrategias conoce para resolver un problema de matemática, más se demora en determinar su solución

\begin{tabular}{lll}
\hline Alternativas & Frecuencia & Porcentaje \\
\hline Total, acuerdo & 0 & 0,0 \\
Bastante de acuerdo & 10 & 25,0 \\
Bastante en desacuerdo & 8 & 20,0 \\
Total, desacuerdo & 25 & 50,0 \\
Ninguna & 2 & 5,0 \\
Total & 45 & 100 \\
\hline
\end{tabular}


En la octava pregunta, el $80 \%$ de los estudiantes está en total acuerdo que si tiene buenos conocimientos matemáticos podrá resolver fácilmente problemas aplicados, el 10\% está bastante desacuerdo, mientras que el $10 \%$ restante no está ni de acuerdo, ni en desacuerdo, datos que se refleja en la tabla 8 .

\section{Tabla 8}

Si se tienen buenos conocimientos matemáticos se podrá resolver fácilmente problemas aplicados

\begin{tabular}{lll}
\hline Alternativas & Frecuencia & Porcentaje \\
\hline Total, acuerdo & 40 & 80,0 \\
Bastante de acuerdo & 0 & 0,0 \\
Bastante en desacuerdo & 0 & 0,0 \\
Total, desacuerdo & 0 & 0,0 \\
Ninguna & 5 & 10,0 \\
Total & 45 & 100 \\
\hline
\end{tabular}

En la novena pregunta, el $70 \%$ de los estudiantes está en total acuerdo que su capacidad para inducir fórmulas y resolver problemas de matemática, es fundamental para su proceso de resolución, el 20\% está en total desacuerdo, mientras que el 10\% restante no está ni de acuerdo, ni en desacuerdo, datos que se refleja en la tabla 9.

\section{Tabla 9}

La capacidad para inducir formulas y resolver problemas de matemática, es fundamental para el proceso de resolución

\begin{tabular}{lll}
\hline Alternativas & Frecuencia & Porcentaje \\
\hline Total, acuerdo & 35 & 70,0 \\
Bastante de acuerdo & 0 & 0,0 \\
Bastante en desacuerdo & 0 & 0,0 \\
Total, desacuerdo & 6 & 20,0 \\
Ninguna & 4 & 10,0 \\
Total & 45 & 100 \\
\hline
\end{tabular}

En la décima pregunta, el $63 \%$ de los estudiantes está bastante de acuerdo que su capacidad de análisis lo favorece en la resolución de problemas, el 25\% está en bastante desacuerdo, mientras que el $12 \%$ restante no está ni de acuerdo, ni en desacuerdo, datos que se refleja en la tabla 10.

Tabla 10

La capacidad de análisis favorece la resolución de problemas

\begin{tabular}{lll}
\hline Alternativas & Frecuencia & Porcentaje \\
\hline Total, acuerdo & 0 & 0,0 \\
Bastante de acuerdo & 30 & 63,0 \\
Bastante en desacuerdo & 9 & 25,0 \\
Total, desacuerdo & 0 & 0,0 \\
Ninguna & 6 & 12,0 \\
Total & 45 & 100 \\
\hline
\end{tabular}




\section{Hábitos de estudio para la Matemática}

En la décima primera pregunta, el $85 \%$ de los estudiantes está en total acuerdo que procura ampliar los conocimientos adquiridos en clase, mientras que el $15 \%$ está bastante de acuerdo, datos que se refleja en la tabla 11.

\section{Tabla 11}

Interés de ampliar los conocimientos adquiridos en clase

\begin{tabular}{lll}
\hline Alternativas & Frecuencia & Porcentaje \\
\hline Total, acuerdo & 38 & 85,0 \\
Bastante de acuerdo & 7 & 15,0 \\
Bastante en desacuerdo & 0 & 0,0 \\
Total, desacuerdo & 0 & \\
Ninguna & 0 & 0,0 \\
Total & 45 & 100 \\
\hline
\end{tabular}

En la décima segunda pregunta, el 60\% de los estudiantes está bastante de acuerdo que la resolución de problemas de la Matemática Académica, permite construir y conceptualizar conocimientos de Matemática Superior, el 25\% está en bastante desacuerdo, mientras que el $15 \%$ restante no está ni de acuerdo, ni en desacuerdo, datos que se refleja en la tabla 12.

\section{Tabla 12}

La resolución de problemas de la Matemática Académica permite construir y conceptualizar conocimientos de Matemática Superior

\begin{tabular}{lll}
\hline Alternativas & Frecuencia & Porcentaje \\
\hline Total, acuerdo & 0 & 0,0 \\
Bastante de acuerdo & 27 & 60,0 \\
Bastante en desacuerdo & 11 & 25,0 \\
Total, desacuerdo & 0 & \\
Ninguna & 7 & 15,0 \\
Total & 45 & 100 \\
\hline
\end{tabular}

En la décima tercera pregunta, el 50\% de los estudiantes está bastante de acuerdo que es fundamental la fuerza de voluntad de la o él estudiante para estimular a realizar un esfuerzo orientado a aprender conocimientos sólidos de Matemática Superior, el 30\% está en bastante desacuerdo, el 10\% está en total acuerdo, mientras que el $10 \%$ restante no está ni de acuerdo, ni en desacuerdo, datos que se refleja en la tabla 13.

Tabla 13

La fuerza de voluntad del estudiante estimular el esfuerzo para aprender conocimientos sólidos de Matemática Superior

\begin{tabular}{lll}
\hline Alternativas & Frecuencia & Porcentaje \\
\hline Total, acuerdo & 5 & 10,0 \\
Bastante de acuerdo & 22 & 50,0 \\
Bastante en desacuerdo & 13 & 30,0 \\
Total, desacuerdo & 0 & 0,0 \\
Ninguna & 5 & 10,0 \\
Total & 45 & 100 \\
\hline
\end{tabular}


En la décima cuarta pregunta, el 50\% de los estudiantes está bastante de acuerdo que, para interpretar, resolver y evaluar resultados de un modelo matemático, son suficientes los conocimientos de la Matemática Académica, el 30\% está en bastante desacuerdo, mientras que el $20 \%$ restante no está ni de acuerdo, ni en desacuerdo, datos que se refleja en la tabla 14.

\section{Tabla 14}

Para interpretar, resolver y evaluar resultados de un modelo matemático son suficientes los conocimientos de la Matemática Académica

\begin{tabular}{lll}
\hline Alternativas & Frecuencia & Porcentaje \\
\hline Total, acuerdo & 0 & 0,0 \\
Bastante de acuerdo & 22 & 50,0 \\
Bastante en desacuerdo & 13 & 30,0 \\
Total, desacuerdo & 0 & 0,0 \\
Ninguna & 10 & 20,0 \\
Total & 45 & 100 \\
\hline
\end{tabular}

En la décima quinta pregunta, el $60 \%$ de los estudiantes está bastante de acuerdo que, la resolución de problemas de la Matemática Aplicada permite explicar, integrar y emplear conceptos de Matemática Superior, el 30\% está en total desacuerdo, mientras que el 10\% restante no está ni de acuerdo, ni en desacuerdo, datos que se refleja en la tabla 15.

\section{Tabla 15}

La resolución de problemas de la Matemática Aplicada permite explicar, integrar y emplear conceptos de Matemática Superior

\begin{tabular}{lll}
\hline Alternativas & Frecuencia & Porcentaje \\
\hline Total, acuerdo & 0 & 0,0 \\
Bastante de acuerdo & 27 & 60,0 \\
Bastante en desacuerdo & 0 & 0,0 \\
Total, desacuerdo & 13 & 30,0 \\
Ninguna & 5 & 10,0 \\
Total & 45 & 100 \\
\hline
\end{tabular}

En la décima sexta pregunta, el 50\% de los estudiantes está bastante de acuerdo que valora la resolución de problemas de la Matemática Aplicada, el 20\% está en bastante desacuerdo, el $10 \%$ está en total acuerdo, otro $10 \%$ está en total desacuerdo, mientras que el $10 \%$ restante no está ni de acuerdo, ni en desacuerdo, datos que se refleja en la tabla 16.

\section{Tabla 16}

Se valora la resolución de problemas de la Matemática Aplicada

\begin{tabular}{lll}
\hline Alternativas & Frecuencia & Porcentaje \\
\hline Total, acuerdo & 7 & 10,0 \\
Bastante de acuerdo & 22 & 50,0 \\
Bastante en desacuerdo & 9 & 20,0 \\
Total, desacuerdo & 7 & 10,0 \\
Ninguna & 0 & 0,0 \\
Total & 45 & 100 \\
\hline
\end{tabular}


En la décima séptima pregunta, el 65\% de los estudiantes está bastante de acuerdo que, para una adecuada valoración de la Matemática Superior es necesario la resolución de problemas y modelos matemáticos, el $25 \%$ está en bastante desacuerdo, mientras que el $10 \%$ restante no está ni de acuerdo, ni en desacuerdo, datos que se refleja en la tabla 17.

Tabla 17

Para una adecuada valoración de la Matemática Superior es necesario la resolución de problemas y modelos matemáticos

\begin{tabular}{lll}
\hline Alternativas & Frecuencia & Porcentaje \\
\hline Total, acuerdo & 0 & 0,0 \\
Bastante de acuerdo & 29 & 65,0 \\
Bastante en desacuerdo & 11 & 25,0 \\
Total, desacuerdo & 0 & 0,0 \\
Ninguna & 5 & 10,0 \\
Total & 45 & 100 \\
\hline
\end{tabular}

En la décima octava pregunta, el 50\% de los estudiantes está bastante de acuerdo que memoriza sin analizar lo que aprendió, el $40 \%$ está en total desacuerdo, mientras que el $10 \%$ restante no está ni de acuerdo, ni en desacuerdo, datos que se refleja en la tabla 18.

Tabla 18

El estudiante memoriza sin analizar lo que aprendió

\begin{tabular}{lll}
\hline Alternativas & Frecuencia & Porcentaje \\
\hline Total, acuerdo & 0 & 0,0 \\
Bastante de acuerdo & 22 & 50,0 \\
Bastante en desacuerdo & 0 & 0,0 \\
Total, desacuerdo & 18 & 40,0 \\
Ninguna & 5 & 10,0 \\
Total & 45 & 100 \\
\hline
\end{tabular}

En la décima novena pregunta, el 50\% de los estudiantes está en total desacuerdo que no es necesario que se estimule un esfuerzo para aprender conocimientos de la Matemática Superior y sus aplicaciones, el 30\% está bastante de acuerdo, el 10\% está en bastante desacuerdo, mientras que el $10 \%$ restante no está ni de acuerdo, ni en desacuerdo, datos que se refleja en la tabla 19.

\section{Tabla 19}

No es necesario que se estimule un esfuerzo para aprender conocimientos de la Matemática Superior y sus aplicaciones

\begin{tabular}{lll}
\hline Alternativas & Frecuencia & Porcentaje \\
\hline Total, acuerdo & 0 & 0,0 \\
Bastante de acuerdo & 13 & 30,0 \\
Bastante en desacuerdo & 5 & 10,0 \\
Total, desacuerdo & 22 & 50,0 \\
Ninguna & 5 & 10,0 \\
Total & 45 & 100 \\
\hline
\end{tabular}


En la vigésima pregunta, el 50\% de los estudiantes está bastante de acuerdo que elabora esquemas, gráficos, para el análisis matemático, el 35\% está en bastante desacuerdo, mientras que el $15 \%$ restante no está ni de acuerdo, ni en desacuerdo, datos que se refleja en la tabla 20.

\section{Tabla 20}

Se elabora esquemas, gráficos, para el análisis matemático

\begin{tabular}{lll}
\hline Alternativas & Frecuencia & Porcentaje \\
\hline Total, acuerdo & 0 & 0,0 \\
Bastante de acuerdo & 22 & 50,0 \\
Bastante en desacuerdo & 16 & 35,0 \\
Total, desacuerdo & 0 & 0,0 \\
Ninguna & 7 & 15,0 \\
Total & 45 & 100 \\
\hline
\end{tabular}

\section{Niveles de confianza del estudiante para la resolución de problemas}

En la vigésima primera pregunta, el $50 \%$ de los estudiantes está bastante de acuerdo que trabajar con la matemática no lo asusta en lo absoluto, el $40 \%$ está en bastante desacuerdo, mientras que el $10 \%$ restante no está ni de acuerdo, ni en desacuerdo, datos que se refleja en la tabla 21.

\section{Tabla 21}

Trabajar con la matemática no asusta al estudiante

\begin{tabular}{lll}
\hline Alternativas & Frecuencia & Porcentaje \\
\hline Total, acuerdo & 0 & 0,0 \\
Bastante de acuerdo & 22 & 50,0 \\
Bastante en desacuerdo & 18 & 40,0 \\
Total, desacuerdo & 0 & 0,0 \\
Ninguna & 5 & 10,0 \\
Total & 45 & 100 \\
\hline
\end{tabular}

En la vigésima segunda pregunta, el $60 \%$ de los estudiantes está bastante de acuerdo que tiene confianza en sí mismo cuando resuelve un problema de Matemática, mientras que el $40 \%$ restante está en bastante desacuerdo, datos que se refleja en la tabla 22.

Tabla 22

El estudiante tiene confianza en sí mismo cuando resuelve un problema de Matemática

\begin{tabular}{lll}
\hline Alternativas & Frecuencia & Porcentaje \\
\hline Total, acuerdo & 0 & 0,0 \\
Bastante de acuerdo & 27 & 60,0 \\
Bastante en desacuerdo & 13 & 40,0 \\
Total, desacuerdo & 0 & 0,0 \\
Ninguna & 0 & 0,0 \\
Total & 45 & 100 \\
\hline
\end{tabular}


En la vigésima tercera pregunta, el 50\% de los estudiantes está bastante de acuerdo que escucha con atención todas las indicaciones del profesor, mientras que el 50\% restante está en bastante desacuerdo, datos que se refleja en la tabla 23.

Tabla 23

El estudiante escucha con atención todas las indicaciones del profesor

\begin{tabular}{lll}
\hline Alternativas & Frecuencia & Porcentaje \\
\hline Total, acuerdo & 0 & 0,0 \\
Bastante de acuerdo & 23 & 50,0 \\
Bastante en desacuerdo & 22 & 50,0 \\
Total, desacuerdo & 0 & \\
Ninguna & 0 & 0,0 \\
Total & 45 & 100 \\
\hline
\end{tabular}

En la vigésima cuarta pregunta, el $60 \%$ de los estudiantes está bastante de acuerdo que no se altera cuando tiene que trabajar en la resolución de ejercicios basados en problemas, el 30\% está en bastante desacuerdo, mientras que el 10\% restante no está ni de acuerdo, ni en desacuerdo, datos que se refleja en la tabla 24.

\section{Tabla 24}

El estudiante no se altera cuando tiene que trabajar en la resolución de ejercicios basados en problemas

\begin{tabular}{lll}
\hline Alternativas & Frecuencia & Porcentaje \\
\hline Total, acuerdo & 0 & 0,0 \\
Bastante de acuerdo & 27 & 60,0 \\
Bastante en desacuerdo & 13 & 30,0 \\
Total, desacuerdo & 0 & 0,0 \\
Ninguna & 5 & 10,0 \\
Total & 45 & 100 \\
\hline
\end{tabular}

En la vigésima quinta pregunta, el $60 \%$ de los estudiantes está en bastante desacuerdo que le provoca gran satisfacción resolver ejercicios basados en problemas, mientras que el $40 \%$ restante está en bastante acuerdo, datos que se refleja en la tabla 25.

Tabla 25

Satisfacción por resolver ejercicios basados en problemas

\begin{tabular}{lll}
\hline Alternativas & Frecuencia & Porcentaje \\
\hline Total, acuerdo & 0 & 0,0 \\
Bastante de acuerdo & 18 & 40,0 \\
Bastante en desacuerdo & 27 & 60,0 \\
Total, desacuerdo & 0 & 0,0 \\
Ninguna & 0 & 0,0 \\
Total & 45 & 100 \\
\hline
\end{tabular}

En la vigésima sexta pregunta, el 50\% de los estudiantes está bastante de acuerdo que la Matemática implica principalmente memorización de conceptos, el $40 \%$ está en bastante desacuerdo, mientras que el 10\% restante no está ni de acuerdo, ni en desacuerdo, datos que se refleja en la tabla 26. 
Tabla 26

La Matemática implica principalmente memorización de conceptos

\begin{tabular}{lll}
\hline Alternativas & Frecuencia & Porcentaje \\
\hline Total, acuerdo & 0 & 0,0 \\
Bastante de acuerdo & 22 & 50,0 \\
Bastante en desacuerdo & 18 & 40,0 \\
Total, desacuerdo & 0 & 0,0 \\
Ninguna & 5 & 10,0 \\
Total & 45 & 100 \\
\hline
\end{tabular}

En la vigésima séptima pregunta, el $60 \%$ de los estudiantes está bastante en desacuerdo que se sienta más motivado a participar en clases realizando ejercicios basados en la resolución de problemas, el 30\% está bastante de acuerdo, mientras que el 10\% restante no está ni de acuerdo, ni en desacuerdo, datos que se refleja en la tabla 27.

Tabla 27

Motivación para participar en clases realizando ejercicios basados en la resolución de problemas

\begin{tabular}{lll}
\hline Alternativas & Frecuencia & Porcentaje \\
\hline Total, acuerdo & 0 & 0,0 \\
Bastante de acuerdo & 13 & 30,0 \\
Bastante en desacuerdo & 27 & 60,0 \\
Total, desacuerdo & 0 & 0,0 \\
Ninguna & 5 & 10,0 \\
Total & 45 & 100 \\
\hline
\end{tabular}

En la vigésima octava pregunta, el $60 \%$ de los estudiantes está bastante en desacuerdo que la enseñanza de la matemática basada en la resolución de problemas hace que el contenido del curso sea más fácil de entender en comparación con una clase tradicional, el 30\% está bastante de acuerdo, mientras que el 10\% restante no está ni de acuerdo, ni en desacuerdo, datos que se refleja en la tabla 28.

\section{Tabla 28}

La enseñanza de la matemática basada en la resolución de problemas hace que el contenido del curso sea más fácil de entender en comparación con una clase

tradicional

\begin{tabular}{lll}
\hline Alternativas & Frecuencia & Porcentaje \\
\hline Total, acuerdo & 0 & 0,0 \\
Bastante de acuerdo & 13 & 30,0 \\
Bastante en desacuerdo & 27 & 60,0 \\
Total, desacuerdo & 0 & 0,0 \\
Ninguna & 5 & 10,0 \\
Total & 45 & 100 \\
\hline
\end{tabular}

En la vigésima novena pregunta, el 100\% de los estudiantes está en total acuerdo que los errores que comete durante la resolución de un problema, deben ser objeto de análisis, datos que se refleja en la tabla 29. 
Tabla 29

Acuerdo en que los errores que comete durante la resolución de un problema, deben ser objeto de análisis

\begin{tabular}{lll}
\hline Alternativas & Frecuencia & Porcentaje \\
\hline Total, acuerdo & 45 & 100,0 \\
Bastante de acuerdo & 0 & 0,0 \\
Bastante en desacuerdo & 0 & 0,0 \\
Total, desacuerdo & 0 & \\
Ninguna & 0 & 0,0 \\
Total & 45 & 100 \\
\hline
\end{tabular}

En la trigésima pregunta, el 50\% de los estudiantes está en total acuerdo que se siente confiado al resolver un problema de la clase utilizando la modelación matemática, mientras que el otro $50 \%$ está bastante en desacuerdo, datos que se refleja en la tabla 30.

\section{Tabla 30}

El estudiante se siente confiado al resolver un problema de la clase utilizando la modelación matemática

\begin{tabular}{lll}
\hline Alternativas & Frecuencia & Porcentaje \\
\hline Total, acuerdo & 23 & 50,0 \\
Bastante de acuerdo & 0 & 0,0 \\
Bastante en desacuerdo & 22 & 50,0 \\
Total, desacuerdo & 0 & 0,0 \\
Ninguna & 0 & 0,0 \\
Total & 45 & 100 \\
\hline
\end{tabular}

\section{Discusión}

Los resultados obtenidos determinan que los conocimientos que los estudiantes de la Carrera de la Pedagogía poseen, contribuyen con la resolución de problemas matemáticos, pues, así lo considera la mayoría de los mismos representada en un $75 \%$. En esta misma línea discursiva, la mayor parte del estudiantado considera que los conocimientos de Matemática Académica inciden en el desarrollo de la Matemática Superior, además que consideran que si tienen buenos conocimientos matemáticos podrán resolver fácilmente problemas aplicados.

En lo que respecta a los hábitos de estudio, estos influyen en la resolución de problemas matemáticos, ya que, en criterio de la mayoría de estudiantes, la ampliación de conocimientos, fuerza de voluntad, esfuerzo para aprender conocimientos y elaboración de esquemas y gráficos son acciones que contribuyen con tal proceso dentro de su formación pedagógica.

Finalmente, la mayor parte de estudiantes sostienen que tienen confianza en sí mismos al resolver un problema matemático, lo que les permite escuchar con atención todas las indicaciones del profesor, además de comprender que los errores que comete durante la resolución de un problema, deben ser objeto de análisis.

\section{Conclusiones}

- Los resultados obtenidos permiten concluir que los conocimientos, hábitos y niveles de confianza influyen en la resolución de problemas matemáticos en los 
estudiantes de la Carrera de la Pedagogía las Ciencias Experimentales de la Matemática y la Física de la Universidad Técnica "Luis Vargas Torres", ubicada en la provincia de Esmeraldas. Esto se debe a que los saberes matemáticos previos que el estudiante posee le permiten abordar de forma estratégica el problema que se necesita solucionar, es decir, constituyen la base teoría para su resolución.

- Por otra parte, los hábitos son acciones que al estudiante le permiten desarrollar habilidades y destrezas en sus procesos de formación, razón por la cual pueden ser utilizados de forma positiva para realizar actividades educativas dentro del contexto de la matemática como la resolución de distintas clases de problemas, contribuyendo así con su propio aprendizaje.

- El nivel de confianza es otro aspecto que puede contribuir positivamente en la resolución de problemas matemáticos, ya que si un estudiante se siente calmado y confía en sus capacidades demuestra una actitud positiva que le permitirá desarrollar eficientemente esta actividad en beneficio de su propia formación académica.

\section{Referencias Bibliográficas}

Aliaga, J., Rivera, I., Cahuana, M. y Paico, M. (2014). La construcción del conocimiento escolar matemático en alumnos del conocimiento escolar matemático en alumnos (una mirada desde los niños). REVISTA IIPSI, 17(2), 133-143.

Bahamonde, S. y Vicuña, J. (2011). Resolución de problemas matemáticos. Tesis de pregrado. Punta Arenas: Universidad de Magallanes.

Blanco, L., Cárdenas, J. y Caballero, A. (2015). La resolución de problemas de Matemáticas en la formación inicial de profesores de primaria. Extremadura: Servicio de Publicaciones de la Universidad de Extremadura.

Castaño, A. (2011). Introducción al concepto de hábito de Charles Peirce para el comportamiento del consumidor. Punto de Vista, 3(9), 1-6.

Conejeros, L., Rojas, J. y Segure, T. (2010). Confianza: un valor necesario y ausente en la educación chilena. Perfiles Educativos, 32(129), 30-46.

Donoso, E., Valdés, P., Cisternas, P. y Cáceres, R. (2020). Enseñanza de la resolución de problemas matemáticos: Un análisis de correspondencias múltiples. Diálogos sobre Educación, 11(21), 1-22. doi: https://doi.org/10.32870/dse.v0i21.629

Garcés, H. (2010). Investigación Científica. Quito: Abya Yala.

Huari, N. (2019). Los hábitos de estudio y la resolución de problemas matemáticos según la madurez mental del estudiante en una institución educativa pública. Tesis de doctorado. Lima: Universidad César Vallejo.

Leal, S. y Bong, S. (2015). La resolución de problemas matemáticos en el contexto de los proyectos de aprendizaje. Revista de Investigación, 39(84), 71-93. 
Naghi, M. (s.f.). Metodología de la Investigación (segunda ed.). Limusa Noriega.

Pérez, Y. y Beltrán, C. (2011). ¿Qué es un problema en Matemática y cómo resolverlo? Algunas consideraciones preliminares. EduSol, 11(34), 74-89.

Pérez, Y. y Ramírez, R. (2011). Estrategias de enseñanza de la resolución de problemas matemáticos. Fundamentos teóricos y metodológicos. Revista de Investigación, 73(35), 169-194.

Zamora, J. (2017). Propuesta de método de resolución de problemas matemáticos en educación primaria. Tesis de pregrado. Castellón de la Plana: Universitat Jaume I .

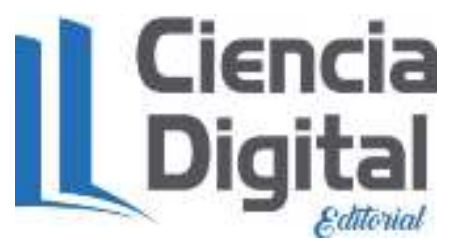




\section{PARA CITAR EL ARTÍCULO INDEXADO.}

Hidalgo Portocarrero, G. E., Merino Córdova, P. A., Estupiñan Cox, B. F., \& Tapia Aguilar, O. E. (2021). Conocimientos, hábitos, niveles de confianza para la resolución de problemas matemáticos en los estudiantes de pedagogía. ConcienciaDigital, 4(4.1), 1938. https://doi.org/10.33262/concienciadigital.v4i4.1.1922

\section{【 Ciencia}

El artículo que se publica es de exclusiva responsabilidad de los autores y no necesariamente reflejan el pensamiento de la Revista Conciencia Digital.

El artículo queda en propiedad de la revista y, por tanto, su publicación parcial y/o total en otro medio tiene que ser autorizado por el director de la Revista Conciencia Digital.

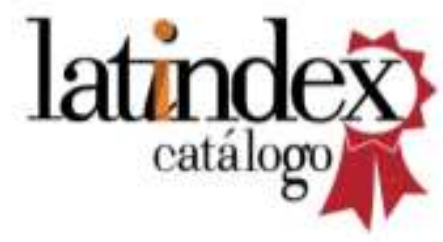

